\title{
Nocardia infection in splenectomized patients: case reports and a review of the literature
}

\author{
E.A. Abdi, J.C. Ding and I.A. Cooper \\ Haematology Research Unit, Peter MacCallum Hospital, Cancer Institute, Melbourne, Australia
}

\begin{abstract}
Summary: Opportunistic infections are increasingly becoming a problem in cancer patients amongst whom infection with Nocardia species is particularly difficult to detect due to the capricious natural history of the disease. Three cases of Nocardia infection in patients who had undergone splenectomy for haematological malignancy are presented. These cases illustrate the diverse mode of presentation, the natural history and the difficulties in early and accurate diagnosis of Nocardia infection. Despite the difficulties in arriving at the correct diagnosis, these cases highlight the importance of early institution of appropriate antibiotic therapy. Antibiotics should be given in adequate doses to control the initial infection and be maintained for a prolonged period to prevent relapses.
\end{abstract}

\section{Introduction}

In recent years significant advances have been made in the recognition and therapy of opportunistic infections in cancer patients and other immuno-compromised patients. Most often such opportunistic infections are of bacterial aetiology. Among the organisms responsible, Nocardia species are being reported with increasing frequency. ${ }^{1,2}$ We describe three patients with haematological malignancies who developed Nocardia infections after splenectomy. These cases illustrate the diverse mode of presentation and the difficulties in early and accurate diagnosis of Nocardia infection.

\section{Cases}

\section{Case 1}

A 43 year old male presented in July 1972 with stage III A, nodular poorly differentiated lymphocytic lymphoma. Soon after completing radiation therapy he developed splenomegaly and pancytopenia requiring splenectomy for hypersplenism. Two years later, his lymphoma relapsed requiring chemotherapy for 2

Correspondence and present address: E.A. Abdi, M.B., B.S., F.R.A.C.P., Department of Medicine, Cross Cancer Institute, 11560 University Avenue, Edmonton, Alberta, Canada, T6G 1Z2, (403) 432-8717

Accepted: 12 November 1986 years. Throughout this 2 year period there were intermittent episodes of cough occasionally productive of purulent sputum, but no organisms could be isolated. In October 1976 in addition to fever and a productive cough, chest $\mathrm{X}$-rays revealed widespread ill-defined nodular opacities throughout both lung fields. Sputum examination and other microbiological surveys were negative. With oral amoxycillin there was a rapid clinical and radiological resolution. In January 1977 he presented with a progressive left hemiparesis. Cerebrospinal fluid (CSF) analysis showed no cells or organisms. Augmented computerized axial tomography scans of the head showed a deep-seated small lesion in the right cerebral hemisphere. He improved with whole brain irradiation. A month later he developed a fever, cough productive of purulent sputum, scattered adventitial sounds in the chest and. radiologically, consolidation of right mid- and lower zones. Sputum microscopy showed numerous pus cells and Gram-positive filamentous branching bacilli. Cultures confirmed the organisms as Nocardia asteroides. He was commenced on co-trimoxazole and within a week his chest X-ray showed a rapid but incomplete resolution. His lymphoma however showed further visceral progression leading to his death. At autopsy, the right lung contained an abscess. Microabscesses in other parts of the lung and the kidneys were also present. Nocardia asteroides was cultured from the abscess cavity in the lung and kidney. The lesion present in the brain showed progressive multifocal leucoencephalopathy but no abscess or tumour infiltration was seen. 


\section{Case 2}

A 29 year old female presented with stage IIIB Hodgkin's disease in March 1968 for which she received radiation therapy. Three years later Hodgkin's disease relapsed in the lung parenchyma. She achieved a complete remission with chemotherapy. In March 1972 the spleen became palpable requiring a laparotomy; histologically there was no evidence of tumour infiltrating the spleen. Four years later there was another relapse of Hodgkin's disease. While receiving chemotherapy she developed severe dyspnoea, a cough productive of yellow sputum, and fever. Chest X-rays showed diffuse parenchymal opacification. Sputum microscopy showed pus cells, but no organism could be isolated. Other microbiological surveys were negative. She was empirically treated with oral co-trimoxazole, with rapid clinical improvement. Similar respiratory symptoms recurred intermittently from November 1977 to March 1978 and serial chest $\mathrm{X}$-rays showed intermittent patchy consolidation, sputum cultures showed only pus cells but no organisms. In August 1978 she developed pleuritic chest pain, fever, a productive cough and dyspnoea and partial consolidation of the right lower zone. Sputum cultures were negative. Through fibreoptic bronchoscopy bronchial washings and brush biopsy specimens were obtained which showed Gram-positive filamentous bacilli and Nocardia asteroides organisms were isolated on culture. This organism was isolated from subsequent sputum cultures also. She was started on co-trimoxazole, which was continued for 6 months. No recurrence of respiratory symptoms took place on cessation of co-trimoxazole. She remained relatively well until 1981 when she developed progressive cancer, unresponsive to any chemotherapy agent, and died. At autopsy she had widespread tumour but there was no evidence of Nocardia infection.

\section{Case 3}

A 44 year old male presented in September 1969 with stage IV B nodular lymphoma. Between 1969 and 1978 lymphoma relapsed several times requiring treatment with chemotherapy. In May 1979 he developed massive splenomegaly with pancytopenia and underwent a splenectomy. Within 3 months of splenectomy he developed a productive cough, dyspnoea, fever, rigors and chest X-rays showed right mid-zone consolidation. Repeated sputa, blood and other available body fluid cultures were all negative. Such episodes of fever, rigors, pleuritic chest pain, cough and dyspnoea subsequently recurred every 4-6 weeks, lasting 2-5 days at a time. These episodes remained undiagnosed until January 1980, when, apart from previous symptoms, he also developed non-tender skin and subcutaneous nodules on his trunk and extremities. Chest
$\mathrm{X}$-rays again showed opacification and consolidation of the right mid-zone. Pus drained from skin nodules showed branching filamentous Gram-positive bacteria and cultures confirmed the presence of Nocardia asteroides. He was started on co-trimoxazole with prompt resolution of symptoms, skin nodules and the opacity in the right mid-zone. In June 1980 he had a recurrence of respiratory symptoms and signs. Sputum cultures were negative. Co-trimoxazole was empirically commenced with a resolution of symptoms and radiological abnormality. During the period of maintenance therapy with co-trimoxazole the patient developed clinical irido-cyclitis. A biopsy of iris showed non-specific inflammatory change but there was no other evidence of Nocardia infection. Fever, cough and other symptoms recurred 4 months after stopping co-trimoxazole. Microbiological surveys were negative. Co-trimoxazole was recommenced with a resolution of symptoms. During the next 2 years he had several similar episodes and although Nocardia organisms have not been isolated on any further occasion, the symptoms resolved each time co-trimoxazole was instituted when other antibiotics failed to resolve symptoms.

\section{Discussion}

The above three cases illustrate the diverse mode of presentation of Nocardia infections, and the difficul ties in its early and accurate diagnosis. Case 3 also demonstrates the relapsing nature of Nocardia asteroides infection. In the three cases reported above, infection with Nocardia species occurred only after splenectomy. Although the underlying tumour was still present and immunosuppressive agents were being used, the infection did not occur while the patient had an intact spleen. In our institute, we have not encountered Nocardia infection in any patient with an intact spleen.

Nocardia infection is often difficult to diagnose as it produces a wide spectrum of diseases with few specific symptoms and there are no pathognomonic clinical, laboratory or radiological features. ${ }^{3,4}$ It often mimics other more familiar illnesses, especially neoplastic disorders, and may co-exist with other diseases which are easier to diagnose. Microbiological techniques for this infection remain somewhat ill-defined; isolating the organism often requires prolonged incubation. ${ }^{5} \mathrm{~A}$ greater prevalence of Nocardia infection in immunocompromised patients, ${ }^{2}$ in patients with neoplastic diseases, ${ }^{6}$ and in patients with organ transplantation has been reported. ${ }^{4}$ However, Nocardia infection in the absence of any underlying disorder is also well recognized.' Even the isolation of Nocardia organisms from a patient may not necessarily equate with infection due to Nocardia. ${ }^{7,8}$ Nocardia species are 
sometimes saprophytes in the upper airways. However, most microbiologists believe that isolation of Nocardia organisms from patients should be viewed as infection and not as contamination., 9

Nocardia asteroides causes more infections than other members of the genus. ${ }^{2}$ The infection is usually chronic but it can be acute with rapid dissemination (case 1$)^{3,10}$ particularly in immunosuppressed patients. ${ }^{6}$ Experimental evidence shows that Nocardia organisms are phagocytosed but continue to grow within macrophages, suggesting that the organism is a facultative intracellular parasite., "Intact cell-mediated immunity is the most important defence against nocardial infection. In experiments with laboratory animals a higher rate of infection is observed if phagocytosis is impaired by coating Nocardia organisms with agar or mucin. ${ }^{11}, 12$ If mice have been previously infected, a degree of immunity, believed to be cell-mediated, is observed. ${ }^{11,}{ }^{13}$ Alveolar macrophages are the first line of pulmonary defence. If an organism survives phagocytosis by macrophage it can establish a focus of infection. Components of cell walls of Nocardia organisms, like mycobacterial cell walls, may even neutralize the macrophage system." The lungs are affected in about $70 \%$ of patients in the form of focal pneumonitis (as in case 2), multiple nodules, miliary abscesses or cavitation and spread can even occur through the pleura to form an empyema. ${ }^{10}$ Haematogenous dissemination occurs in approximately half of patients following pulmonary infection.' Up to $30 \%$ of patients develop brain abscesses. We suspected brain abscess in case 1 but could not confirm it. Skin infections in the form of mycetoma (as in case 3) occur in about $20 \%$ of patients. Sinuses involving bones and subcutaneous tissue may form from mycetomas. The eyes have been reported as a site of Nocardia infection in about $3 \%$ of patients. Patient 3 developed irido-cyclitis but Nocardia organisms could not be isolated at biopsy. Disseminated nocardiosis is seen in about $23-45 \%$ of cases and in those may involve virtually any organ of the body. $1,3,14$

The importance of the spleen in resisting infection has been recognized since 1919. In animal studies, rats which underwent splenectomy prior to infection with bacteria had a higher mortality rate than controls. ${ }^{15}$

The spleen helps develop B- and T-lymphocyte memory and provides helper $\mathrm{T}$-cell function in the production of antibody. The spleen may also protect against some infections by aiding in induction of cellmediated immunity. ${ }^{16}$ After splenectomy the risk of developing fulminant opportunistic infection and potentially lethal septicaemia is greatly enhanced. ${ }^{16,17}$ Most serious post-splenectomy infections and about $80 \%$ of fatal cases occur within 2 to 3 years after splenectomy. ${ }^{18,19}$ Our patients developed Nocardia infection
8 months, 4 years and 5 years after splenectomy. The risk of post-splenectomy infection is greater if splenectomy is performed for haematological malignancies (1 to $25 \%$ ), as in all of our cases, than if splenectomy is performed for trauma $(0.05$ to $1 \%) .{ }^{19}$ Management of post-splenectomy infection involves documentation of cultures from sputa, urine, cerebrospinal fluid, peripheral blood, pleural or ascitic fluid (if present) followed by prompt therapy with appropriate antimicrobial agent(s) in adequate dosages.

Sulphonamides have been the mainstay of treatment for nocardiosis. Addition of trimethoprim to sulphamethoxazole (co-trimoxazole) reduces the mean inhibitory concentration of the latter, which is equivalent to increasing the dose of sulphonamide. ${ }^{20}$ The synergism of this combination depends on the strain of Nocardia, the duration of incubation and the ratio of the two drugs. Our patients responded very well to co-trimoxazole given in a standard dose $(8$ tablets, each containing $80 \mathrm{mg}$ trimethoprim and $400 \mathrm{mg}$ sulphamethoxazole, daily in divided dose). If used alone, the suggested therapeutic dose of sulphonamide is 4 to $8 \mathrm{~g} / \mathrm{day}$, to achieve a peak serum level of 12 to $15 \mathrm{mg} / \mathrm{dl} .^{21}$ If co-trimoxazole or sulphonamides cannot be used due to allergy to sulphonamides or other cause, a combination of ampicillin/amoxycillin and erythromycin has been found to be synergistic in inhibiting the growth of $85 \%$ of Nocardia asteroides strains. ${ }^{22}$ To prevent relapses antibiotics should be continued for at least 3 months and up to one year. ${ }^{3}$ Treatment is further aided by the proper drainage of any localized abscess; this applies particularly to patients with brain abscesses. In the pre-antibiotic era, mortality from nocardiosis was in excess of $75 \%$, whereas currently, survival in excess of $75 \%$ should be expected. ${ }^{1,8}$ The mortality rate increases in (i) patients receiving corticosteroids or antineoplastic agents, (ii) if duration of symptoms with visceral nocardiosis is less than 3 months and (iii) the presence of disseminated infection involving two or more non-contiguous organs. However, even if the patient is receiving immunosuppressive agents, cure of nocardiosis is possible if the disease is mainly pleuropulmonary and treatment is instituted early, ${ }^{14}$ as in cases 2 and 3. Nocardia brain abscess, however, has a poor prognosis unless the abscess is drained early and aggressive antimicrobial therapy initiated.

In summary, the most helpful factor in diagnosing nocardiosis is a high index of suspicion, especially in immunocompromised, splenectomized patients or patients with cancer, who present with symptoms of pyrexia of unknown origin, acute, chronic or recurrent pulmonary infection or if chest X-rays show pulmonary parenchymal changes for which no other cause is apparent or if the patient fails to respond to therapy for other causes. The presence of cutaneous lesion and central nervous system abnormalities should further 
alert one to the possibility of Nocardiosis. There are no pathognomonic clinical, laboratory or radiological signs of Nocardia infection. To make a diagnosis, Nocardia must be suspected, the organism isolated and identified on specific fungal or mycobacterial media. If the diagnosis is still in doubt more aggressive procedures, such as pulmonary brush biopsy, percutaneous lung aspiration needle biopsy or even open lung biopsy must be employed. The importance of Nocardia infection responding satisfactorily to sul-

\section{References}

1. Palmer, D.L., Harvey, R.L. \& Wheeler, J.K. Diagnostic and therapeutic considerations in Nocardia asteroides infection. Medicine (Baltimore) 1974, 53: 391-401.

2. Beaman, B.L., Burnside, J., Edwards, B. \& Causey, W. Nocardial infections in the United States 1972-1974. J Infect Dis 1976, 134: 286-289.

3. Curry, W.A. Human nocardiosis: A clinical review with selected case reports. Arch Int Med 1980, 140: 818-826.

4. Simpson, G.L., Stinson, E.B., Egger, M.J. \& Remington, J.S. Nocardial infections in the immunocompromised host: A detailed study in a defined population. Rev Infect Dis 1981, 3: 492-507.

5. Goodman, J.S. \& Koenig, M.G. Nocardia infections in a general hospital. Ann NY Acad Sci 1970: 552-567.

6. Young, L.S., Armstrong, D., Blevins, A. \& Lieberman, P. Nocardia asteroides infection complicating neoplastic disease. Am J Med 1971, 50: 356-367.

7. Hosty, T.S., McDurmont, C., Ajello, L., George, L.K., Brumfield, G.L. \& Calix, A.A. Prevalence of Nocardia asteroides in sputa examined by a tuberculosis diagnostic laboratory. J Lab Clin Med 1961, 58: 107-114.

8. Rosett, W. \& Hodges, G.R. Recent experiences with nocardial infection. Am J Med Sci 1978, 276: 279-285.

9. Lerner, G., Tarasidis, G.C. \& Jenney, F.S. Nocardiosis, the importance of early diagnosis and treatment. Am Rev Respir Dis 1967, 96: 494-500.

10. Weed, L.A., Anderson, H.A., Good, C.A. \& Baggenstoss, A.H. Nocardiosis: Clinical, bacteriological and pathological aspects. $N$ Engl J Med 1955, 253: 1137-1143.

11. Goodfellow, M.,Brownell, G.H., Serrano, S.A. (ed) In The Biology of Nocardia. Academic Press, New York, 1976, pp 386-408. phonamide alone, co-trimoxazole or other appropriate antibiotic therapy cannot be overemphasized. Any failure to institute appropriate therapy may result in unnecessary morbidity, and mortality from this infection is still common.

\section{Acknowledgement}

We wish to acknowledge Susan Skelton for preparing this manuscript.

12. Williams, D.M., Krick, J.A. \& Remington, J.S. Pulmonary infection in the compromised host. Part 1. Am Rev Respir Dis 1976, 114: 359-369.

13. Krick, J.A. \& Remington, J.S. Resistance to infection with Nocardia asteroides. J. Infect Dis 1975, 135: 665672.

14. Geiseler, P.J. \& Anderson, B.R. Results of therapy in systemic nocardiosis. Am J Med Sci 1979, 278: 188-194.

15. Morris, D.H. \& Bullock, F.A. The importance of spleen in resistance to infection. Ann Surg 1919, 70: 513-521.

16. Francke, E.L. \& Neu, H.C. Post splenectomy infection. Surg Clin North Am 1981, 61: 135-155.

17. Donaldson, S.S., Glatstein, E. \& Vosti, K.L. Bacterial infections in pediatric Hodgkin's disease: Relationship to radiotherapy, chemotherapy and splenectomy. Cancer 1978, 41: 1949-1958.

18. Ellison, E.C. \& Fabri, P.J. Complications of splenectomy: Etiology, prevention and management. Surg Clin North Am 1983, 63: 1313-1330.

19. Mitchell, A. \& Morris, P.J. Surgery of the spleen. Clin Haematol 1983, 12: 565-590.

20. Pavillard, E.R. Treatment of nocardial infection with trimethoprim-sulphamethoxazole. Med J Aust 1973, 1 (Suppl): 65-69.

21. Hoeprich, P.D. Parenchymal respiratory infections. In Hoeprich, P.D. (ed) Infectious Diseases. Harper and Row, Hagerstown , 1977, p 178, 359.

22. Bach, M.C. The chemotherapy of infections due to nocardia. Int J Clin Pharmacol 1975, 11: 283-285. 